\title{
Quasi 9 and 30-40 days periodicities in the solar differential rotation
}

\author{
J. Javaraiah \\ Indian Institute of Astrophysics, Bangalore - 560034, India \\ Tel: 91 (80) 25530672, Fax: 91 (80) 25534043
}

\begin{abstract}
Using the daily Mt. Wilson Doppler velocity data during 1986-1994 (solar cycle 22), we studied the short-term variations of the order of a few days to a month timescales in the solar differential rotation. We represent the differential rotation in the form: $\omega(\lambda)=\bar{A}+\bar{B}\left(5 \sin ^{2} \lambda-1\right)+\bar{C}\left(21 \sin ^{4} \lambda-\right.$ $\left.14 \sin ^{2} \lambda+1\right)$, using a set of Gegenbauer polynomials, where $\omega(\lambda)$ is the angular velocity at latitude $\lambda$. The coefficients $\bar{A}, \bar{B}$, and $\bar{C}$ are free of crosstalk. We found that $\approx 9$-day periodicity is statistically highly significant in the variations of $\bar{C}$ at the maximum of solar cycle 22. A similar periodicity is found in the variations of $\bar{B}$ during the descending phase of the cycle 22 with significant on $\geq 99.9 \%$ confidence level. At this cycle maximum, a $30-$ 40 day periodicity is found to be dominant among the variations in $\bar{B}$, and this periodicity is found in $\bar{A}$ during almost throughout the period $1986-$ 1994. The $\approx 9$-day periodicity in the variation of the differential rotation approximately matches with the known quasi 10-day periodicity in the total solar irradiance (TSI) variability. Hence, we speculate that there exists a relationship between the differential rotation and TSI variability. We suggest that the 9-10 day periodicities of the differential rotation and TSI have a relationship with the production and the emergence rates of the large-scale solar magnetic flux.
\end{abstract}

Keywords: solar rotation, solar magnetic field, solar activity

Email address: jj@iiap.res.in (J. Javaraiah) 


\section{Introduction}

Besides the well known $\approx 11$-year solar cycle, solar activity varies on many short-time scales (a few minutes to a few years) and many long time scales (a few decades to the Sun's evolution time scale). It has been generally accepted that interactions of the Sun's differential rotation (DR) and the solar magnetic field play a basic role in generation of solar activity. However, the exact role of the DR in the variations of the solar activity is not known and the mechanism of solar cycle is not yet fully understood. Therefore, studies on the temporal variations of the DR are important for understanding the role of the DR in the solar variability. So far many attempts have been made to determine the varitions in the DR and detected several periodicities in the DR by using the different data and different techniques (e.g., Howard \& LaBonte, 1980; LaBonte \& Howard, 1982; Gilman \& Howard,, 1984; Balthasar. Vázquez. \& Wöhl, 1986; Komm. Howard. \& Harvev, 1993; Javaraiah \& Gokhale, 1995; Javaraiah \& Komm, 1999; Gupta. Sivaraman, \& Howard, 1999; Howe et al., 2000a; Javaraiah, 2003, 2005; Javaraiah. Bertello. \& Ulrich, 2005; Braiša. Ruždiak, \& Wöhl, 2006; Javaraiah \& Ulrich, 2006; Tlatov, 2007; Javaraiah et al., 2009; Chandra, Vats, \& Iyer, 2010). A higher rotation rate during the cycle minimum than during the maximum is found in many studies (e.g., Brajša, Ruždjak, \& Wöhl, 2006).

Variation of the DR with the 11-year solar cycle is well established now. The 11-year torsional oscillations were discovered by Howard \& LaBonte (1980), using Mt. Wilson Doppler velocity measurements have been confirmed using different data sets and methods (see Javaraiah \& Gokhale, 2002). The torsional oscillations consist of alternating bands of faster and slower than average rotation moving from high latitudes toward the equator. The faster than average bands start at mid-latitudes of about $45^{\circ}$ during the minimum phase of the solar cycle and move equatorward with the rising phase of the new cycle. The bands extend over about $10^{\circ}$ in latitude. The faster than average rotation band is located on the equatorward side of the magnetic activity belt and a slower than average rotation band is located on its poleward side. Thus, it is thought that the torsional oscillations are associated with magnetic shear. At high latitudes, the rotation changes from slower to faster than average at (or just after) the solar maximum, which might be related to the polarity reversal of polar magnetic fields, and remains faster than average to the next solar minimum without clear migratory character. Helioseismic observations show that the tor- 
sional oscillations are not just a superficial phenomenon but they extend to at least the upper third of the solar convection zone (Howe et al., 2000b; Antia, Basu, \& Chitre, 2008). Variations on the few other time scales in the coefficients of solar differential rotation (Javaraiah \& Gokhale, 1995, 1997; Javaraiah \& Komm, 1999; Javaraiah. Bertello, \& Ulrich, 2005) and the residual rotation (Brajša, Ruždjak, \& Wöhl, 2006), including one which is approximately equal to the Gleissberg cycle, have been found using sunspot group data.

It is known that the total solar irradiance (TSI) varies by about $0.1 \%$ over the solar cycle. A number of statistical models of TSI variability have been constructed on the basis of inhomogeneities of surface magnetic field. These models helped to identify the surface magnetic structures (sunspots and faculae) responsible for variations in TSI and also provided widely accepted theoretical explanations. However, the exact mechanism behind the TSI variation is not known (Foukal, 1992; Kuhn, Lin, \& Coulter, 1999; Solanki, Krivova, \& Wenzler, 2005; Domingo et al., 2009).

Kuhn, Libbrecht, \& Dicke (1988) found that a significant part of the solar cycle variation in TSI is a result of the temporal changes in the latitudedependent surface temperature of the Sun. These authors speculated that the DR (turbulent Reynolds tresses and their perturbations) has a role in TSI variability. Besides the $\approx 11$-year periodicity, TSI also seems to vary on timescales which are much shorter than 11-year. For example, a $\approx 10$-day periodicity is found to be prominent in the short variations of TSI (Foukal, 1992; Nikonova, Klochek, \& Palamrchuk, 1998 ) and the existence of a $\approx 30$-day periodicity in the proxies of TSI is reported (e.g., Pap, Willson, \& Donnelly, 1992). However, the amplitudes of the short variations of TSI depend on the phase of a solar cycle (e.g., Pap, Willson, \& Donnelly, 1992). In the present analysis we have attempted to find the short periodicities in the coefficients of the DR determined from the Mt. Wilson velocity data during 1986-1994, because which may help for understanding the TSI variability.

In the next section we describe the methodology and the data analysis. In Section 3 we present the results, and in Section 4 we present conclusions and briefly discuss them. 


\section{Methodology and data analysis}

The Sun's DR can be determined from full disc velocity data using the traditional polynomial expansion:

$$
\omega(\lambda)=A+B \sin ^{2} \lambda+C \sin ^{4} \lambda,
$$

where $\omega(\lambda)$ is the solar rotation rate at latitude $\lambda$, the parameter $A$ represents the equatorial rotation rate, $B$ and $C$ measure the latitude gradient of the rotation rate with $B$ representing mainly low latitudes and $C$ representing largely higher latitudes.

As pointed out by several authors (see Snodgrass, 1984), due to the nonorthogonality of the fit functions, the coefficients $A, B$, and $C$ have a crosstalk which affects their temporal behavior. Snodgrass \& Howard (1985) used the so-called Gegenbauer Polynomials (Morse \& Feshbach, 1953) as a set of diskorthogonal fit functions with $T_{0}^{1}(\sin \lambda)=1, T_{2}^{1}(\sin \lambda)=5 \sin ^{2} \lambda-1$, and $T_{4}^{1}(\sin \lambda)=21 \sin ^{4} \lambda-14 \sin ^{2} \lambda+1$, which leads to the following expansion:

$$
\omega(\lambda)=\bar{A}+\bar{B}\left(5 \sin ^{2} \lambda-1\right)+\bar{C}\left(21 \sin ^{4} \lambda-14 \sin ^{2} \lambda+1\right) .
$$

The coefficients $\bar{A}, \bar{B}$, and $\bar{C}$ are free of crosstalk, $\bar{A}$ represents the 'rigid body' (or 'mean') component in the rotation, $\bar{B}$ and $\bar{C}$ are the components of the DR. If the polynomial expansion is terminated at $\bar{C}$ (or $\mathrm{C}$ ), the coefficients, $\bar{A}, \bar{B}$, and $\bar{C}$ are related to the $\mathrm{A}, \mathrm{B}$, and $\mathrm{C}$ coefficients as follows:

$$
\bar{A}=A+(1 / 5) B+(3 / 35) C ; \quad \bar{B}=(1 / 5) B+(2 / 15) C ; \quad \bar{C}=(1 / 21) C .
$$

In this case, the temporal variation of $\bar{C}$ is qualitatively identical to that of C (Note: in Equation (6) of Snodgrass \& Ulrich (1990) 1/21 is erroneously typed as $2 / 21$ ).

Earlier, using the daily values of the $A, B, C$ coefficients (cf., Equation (1)) derived from the Mt. Wilson velocity data during the period 1967-1994, Javaraiah \& Komm (1999) determined nearly one-year and more than one year periodicities in the DR. In that early paper the authors had focused mainly on the data obtained during the period 1982-1994, after a major change in the Mt. Wilson spectrograph modification which reduced the instrumental noise (see Howard et al., 1983). Recently, Javaraiah et al. (2009) found the existence of $\mathrm{a} \approx 1$.4-year periodicity in $A$ determined from the Mt. Wilson velocity data during 1986-1995, but they did not find any significant variation in $A$ determined from the data during 1996-2007, which are 
measured from the more stable Mt. Wilson spectrograph instrumentation. However, the 1.3-year periodicity in the variation of the low and the middle latitudes' rotation rate near the base of the convection zone, that was detected by Howe et al. (2000a) from helioseismic measurements during the period 1995-1999, is also found to be not persisting after 2001 (Howe et al., 2007). In fact, in several solar activity phenomena a 1.3-year periodicity has been found to be dominant during the cycle 22 and weak or absent in the later period (see Obridko \& Shelting, 2007).

The time series of $A, B$, and $C$ during 1986-1994 have many data gaps that very in size, $1-18$ days. Since here we have attempted to determine the periodicities of the order of a five days to a month only, hence it is necessary to analyse the daily data which have no gaps or have only a few $1-2$ day gaps. We find that the data in the following time (rotation number) intervals contain only a few (two or three) 1-2 days gaps: $7772-7934$, $8197-8297,8559-8726,8902-9023,9280-9376,9583-9720,10056-10161$, $10375-10514$ and $10731-10893$. The size of each of these intervals is sufficient to determine the short periodicities of a few days to a month time. (In the data during a period which is not included in any one of these intervals there are large number of gaps of different sizes. Therefore, they are unfit to use for the present purpose.) Incidentally, each one of these intervals belongs to one of the years 1986-1994, in chronological order. That is, the years 1986, 1987, ...., 1994 contain 7772-7934 (first interval), 8197-8297 (second interval), .., 10731-10893 (last interval), respectively. By using Equation (3), we converted the $A, B$, and $C$ coefficients to the corresponding coefficients $\bar{A}, \bar{B}$, and $\bar{C}$, respectively. We corrected the time series of each of these coefficients by removing the very large-spikes, i.e., we have removed the values which are $>2.5 \sigma$ level (where $\sigma$ is the standard deviation of the original time series). We filled the gaps in each of these time-series from the values obtained by liner-interpolations. Figures 1 and 2 show these corrected time series and also the corresponding uncorrected time-series. As can be seen in these figures, there are variations of the order of few days to a month time scales in $\bar{A}, \bar{B}$ and $\bar{C}$ during many of the aforementioned time intervals. We computed fast Fourier transform (FFT) power spectra of $\bar{A}$, $\bar{B}$, and $\bar{C}$. The results are presented in the next section. 


\section{Results}

Figures $3-5$ show the FFT power spectra of $\bar{A}, \bar{B}$ and $\bar{C}$ derived from the corrected data (solid curves in Figures 1-2) during each of the reliable time intervals found above. Before computing the FFT, the long-term trend of the order of the length of the time series was removed by subtracting the corresponding leaner-model of the series and a cosine bell function was applied to the first and the last $10 \%$ of the time series (see Brault \& White, 1971). In Table 1 we have given the periodicities in the variations of $\bar{A}, \bar{B}$ and $\bar{C}$, whose levels of significance are $\geq 2 \sigma$ in the respective FFT power spectra. The results in this table (and Figures 3-5) suggest that at the solar cycle maximum (i.e., in the interval 8902-9023 during the year 1989), a $\approx 9$-day periodicity is very pronounced in the variations of $\bar{C}$. In the same time this

periodicity is insignificant/absent in the variations of both $\bar{A}$ and $\bar{B}$, and a 30-40 day periodicity is dominant in the variations of $\bar{B}$. During the decay phase of the cycle 22 (years $1991-1993$ ), there is $\sim 9$-day periodicity in the variations of $\bar{B}$ with $>99 \%$ confidence level. There is a suggestion on the existence of the $30-40$ day periodicity in $\bar{A}$ during almost throughout the period $1986-1994$.

\section{Conclusions and discussion}

By analyzing the Mt. Wilson Doppler velocity data during the period 1986-1994, we find:

1. During the maximum phase of the solar cycle 22 there were highly statistically significant $\approx 9$-day and 30-40 day periodicities in the DR coefficients $\bar{C}$ and $\bar{B}$, respectively.

2 . During the descending phase of cycle 22 the $\approx 9$-day periodicity was stronger in $\bar{B}$ than in $\bar{C}$, the $30-40$ day periodicity was weak in both $\bar{B}$ and $\bar{C}$.

3. Near the minimum of cycle 22 the $30-40$ day periodicity was present and the $\approx 9$-day periodicity was insignificant/absent in $\bar{C}$.

4. In $\bar{A}$ the 9-day periodicity was absent during any phase of the solar cycle 22, whereas a 30-40 day periodicity was present in almost throughout this cycle.

It is difficult to detect variations in the equatorial rotation rate determined from the Doppler velocity measurements because several observational 
and instrumental effects can produce spurious peaks with similar periodicities (e.g., Snodgrass, 1983; Lustig \& Wöehl, 1989; Snodgrass \& Ulrich, 1990; Stenflo, 1990; Ulrich \& Bertello, 1996; Wöhl \& Schmidt, 200). Variations in the solar rotation on shorter time scales are more difficult to detect because of the relatively low amplitudes and intermittent nature of them.

Since 1986 the quality of the Mt. Wilson velocity data is high enough to study substantially short variations in the derived velocity values (Snodgrass, 1992; Ulrich, 1998), hence, the dominant $\approx 9$ day and the $30-40$ day periodicities in $\bar{B}$ and $\bar{C}$, found above, may be really exist in the DR. However, the amplitudes of these periodicities in the DR depend on the phases of the solar cycle.

$\mathrm{A} \approx 10$-day periodicity seems to prominently present in the variation of the TSI (see Foukal, 1992; Nikonova, Klochek, \& Palamrchuk, 1998). Pap. Willson. \& Donnelly (1992) found $\mathrm{a} \approx 30$-day periodicity in the proxies of TSI. Recently, Shapiro et al. (2011) found 13- and 27-day periodicities in the spectral solar irradiance. The $\approx 9$-day and 30-40 day periodicities of $\bar{B}$ and $\bar{C}$ approximately match with that known $\approx 10$-day and $\approx 30$-day periodicities in the TSI variability.

The existence of the common periodicities in the DR and the TSI suggests that the latter may be related to the former as pointed by Kuhn, Libbrecht, \& Dicke (1988). Hence, the $\approx 9$-day and 30-40 day periodicities in the DR, which are detected here, may be of particular importance for understanding the TSI variability.

It may be also necessary to note here that the $\approx 9$ day $^{-1}$ frequency in the DR may be the third harmonic of the Sun's rotation frequency. The $\approx 9$-day and $\approx 30$-day periodicities seem to be predominantly present in the solar coronal holes, solar wind, auroral electron power, and geomagnetic parameters (Temmer, Vršnak, \& Veronig, 2007; Emery et al., 2009). Also, helioseismic studies suggest that global solar oscillation frequency changes on time scale as short as nine days (Tripathy et al., 2007).

Howard \& LaBonte (1981) analysed Mt. Wilson magnetograph data during the period 1967 to mid-1980 and found that the rate at which the magnetic flux appears on the Sun is sufficient to create all the flux that is seen at the solar surface within a period of about 10 days. The magnetic structures of the sunspot groups may rise from near the bottom of the convention zone to the surface in about 10 days (Javaraiah \& Gokhale, 1997). Thus, the 9 -10 day periodicity of the DR and the TSI seems to be related to the production and emergence rate of the large-scale solar magnetic flux. 


\section{Acknowledgments}

The author thanks Dr. R. F. Howard for kindly providing the data and the anonymous referees for the useful comments and suggestions. The author is grateful to Dr. Rudi Komm for the helpful discussion on the preliminary results, at NSO Sac Peak in March 2002, and to Dr. John Leibacher for the financial support.

\section{References}

Antia, H.M., Basu, S., Chitre, S.M. Solar rotation rate and its gradients during Cycle 23, Astrophys. J. 681, 680-692, 2008.

Balthasar, H., Vázquez, M., Wöhl, H. Differential rotation of sunspot groups in the period from 1874 through 1976 and changes of the rotation velocity within the solar cycle, Astron. Astrophys. 155, 87-98, 1986.

Brajsa, R., Ruždjak, D., Wöhl, H. Temporal variations of the solar rotation determined by sunspot groups, Sol. Phys. 237, 365-382, 2006.

Brault, J.W., White, O.R. The Analysis and restoration of Astronomical data via the fast Fourier transform, Astron. Astrophys. 13, 169-189, 1971.

Chandra, S., Vats, H.O., Iyer, K.N. Differential rotation measurement of soft X-ray corona, Mon. Not. Roy. Astron. Soc. 407, 1108-1115, 2010.

Domingo, V., Ermolli, I., Fox, P., Fröhlich, C., Haberreiter, M., Krivova, N., Kopp, G., Schmutz, W., Solanki, S.K., Spruit, H.C., Unruh, Y., Vögler, A. Solar surface magnetism and irradiance on time scales from days to the 11-year cycle, Space Sci. Rev. 145, 337-380, 2009.

Foukal, P. Solar luminosity variation, in: Karen L. Harvey (Ed.) The Solar Cycle, CS-27, Astron. Soc. Pac., San Francisco, pp. 439-449, 1992.

Emery, B.A., Richardson, I.G., Evans, D.S., Rich, F.J. Solar wind structure sources and periodicities of auroral electron power over three solar cycles, J. Atmos. Solar-Terr. Phys. 71, 1157-1175, 2009.

Gilman, P.A., Howard, R. Variations in solar rotation with the sunspot cycle, Astrophys. J. 283, 385-391, 1984. 
Gupta, S.S., Sivaraman, K.R., Howard, R. Measurement of Kodaikanal white-light images - III. Rotation rates and activity cycle variations, Sol. Phys. 188, 225-236, 1999.

Howard, R., LaBonte, B.J. The sun is observed to be a torsional oscillator with a period of 11 years, Astrophys. J. 239, L33-L36, 1980.

Howard, R., LaBonte, B.J. Surface magnetic fields during the solar activity cycle, Sol. Phys. 74, 131-145, 1981.

Howard, R., Boyden, J.E., Bruning, D.H., Clark, M.K., Crist, H.W., LaBonte, B.J. The Mount Wilson magnetograph (Report from a Solar Institute), Sol. Phys. 87, 195-203, 1983.

Howe, R., Christensen-Dalsgaard, J., Hill, F., Komm, R.W., Larsen, R.M., Schou, J., Thompson, M.J., Toomre, J. Deeply penetrating banded zonal flows in the solar convection zone, Astrophys. J. 533, L163-166, 2000a.

Howe, R., Christensen-Dalsgaard, J., Hill, F., Komm, R.W., Larsen, R.M., Schou, J., Thompson, M.J., Toomre, J. Dynamic variations at the base of the solar convection zone 2000a, Sci. 287, 2456-2460, 2000b.

Howe, R., Christensen-Dalsgaard, J., Hill, F., Komm, R., Schou, J., Thompson, M.J., Toomre, J. Temporal variations in solar rotation at the bottom of the convection zone: The current status, Adv. Space Res. 24, 40, 915918, 2007.

Javaraiah, J. Long-term variations in the solar differential rotation, Sol. Phys. $212,23-49,2003$.

Javaraiah, J. Sun's retrograde motion and violation of even-odd cycle rule in sunspot activity, Mon. Not. Roy. Astron. Soc. 362, 1311-1318, 2005.

Javaraiah, J., Gokhale, M.H. Periodicities in the solar differential rotation, surface magnetic field and planetary configurations, Sol. Phys. 158, $173-$ 195, 1995.

Javaraiah, J., Gokhale, M.H. Estimation of the depths of initial anchoring and the rising-rates of sunspot magnetic structures from rotation frequencies of sunspot groups, Astron. Astrophys. 327, 795-799, 1997. 
Javaraiah, J., Gokhale, M.H. (Eds.) The Suns rotation, Nova Science Publishers, New York, 2002.

Javaraiah, J., Komm, R.W. Short-term periodicities of the Sun's 'mean' and differential rotation, Sol. Phys. 184, 41-60, 1999.

Javaraiah, J., Bertello, L., Ulrich, R.K. Long-term variations in solar differential rotation and sunspot activity, Sol. Phys. 232, 25-40, 2005.

Javaraiah, J., Ulrich, R.K. Solar-cycle-related variations in the solar differential rotation and meridional flow: a comparison, Sol. Phys. 237, 245-265, 2006.

Javaraiah, J., Ulrich, R.K., Bertello, L., Boyden, J.E. Search for short-term periodicities in the Sun's surface rotation: a revisit, Sol. Phys. 257, 61-69, 2009 .

Komm, R.W., Howard, R.F., Harvey, J.W. Torsional oscillation patterns in photospheric magnetic features, Sol. Phys. 143, 19-39, 1993.

Kuhn, J.R., Libbrecht, K.G., Dicke, R.H. The surface temperature of the sun and changes in the solar constant, Sci. 242, 908, 1988.

Kuhn, J.R., Lin, H., Coulter, R. What can irradiance measurements tell us about the solar magnetic cycle?, Adv. Space Res. 24, 185-194, 1999.

LaBonte, B.J., Howard, R.F. Solar rotation measurements at Mount Wilson. III - Meridional flow and limb-shift 1982, Sol. Phys. 80, 361-372, 1982.

Lustig, G., Wöhl, H. Solar equatorial plasma rotation from 1983 until 1986, Astron. Astrophys. 218, 299-301, 1989.

Morse, P.M., Feshbach, H. Methods of theoretical physics, Part I, McGrawHill Book Company, New York, Toronto, London, 1953.

Nikonova, M.V., Klochek, N.V., Palamrchuk, L.E. Quasi-10-day and 4-day periodicities in solar irradiance, in: Deubner F.L. et al. (Eds.) New Eyes to See Inside the Sun and Stars, IAU Symp. 185, pp. 119-120, 1998.

Obridko, V.N., Shelting, B.D. Occurrence of the 1.3-year periodicity in the large-scale solar magnetic field for 8 solar cycles, Adv. Space Res. 40, $1006-1014,2007$. 
Pap, J.M., Willson, R.C., Donnelly, R.F. Two-parameter model of total solar irradiance variation over the solar cycle, in: Karen L. Harvey (Ed.) The Solar Cycle, CS-27, Astron. Soc. Pac. San Francisco, pp. 491-502, 1992.

Shapiro, A.V., Rozanov, E., Egorova, T., Shapiro, A. I., Peter, Th., Schmutz, W. Sensitivity of the Earths middle atmosphere to short-term solar variability and its dependence on the choice of solar irradiance data set, J. Atmos. Solar-Terr. Phys. 73, 348-355, 2011.

Snodgrass, H.B. Magnetic rotation of the solar photosphere, Astrophys. J. 270, 288-299, 1983.

Snodgrass, H.B. Separation of large-scale photospheric Doppler patterns, Sol. Phys. 94, 13-31, 1984.

Snodgrass, H.B. Synoptic observations of large scale velocity patterns on the Sun, in: Karen L. Harvey (Ed.) The Solar Cycle, CS-27, Astron. Soc. Pac., San Francisco, pp. 205-240, 1992.

Snodgrass, H.B., Howard, R. Torsional oscillations of low mode, Sol. Phys. 95, $221-228,1985$.

Snodgrass, H.B., Ulrich, R.K. Rotation of Doppler features in the solar photosphere, Astrophys. J. 351, 309-316, 1990.

Solanki, S.K., Krivova, N.A., Wenzler, T. Irradiance models, Adv. in Space Res. 35, 376-383, 2005.

Stenflo, J.O. Time invariance of the sun's rotation rate, Astron. Astrophys. 233, $220-228,1990$.

Temmer, M., Vršnak, B., Veronig, A.M. Periodic Appearance of coronal holes and the related variation of solar wind parameters, Sol. Phys. 241, $371-$ 383, 2007.

Tlatov, A.G. 22-year variations of the solar rotation and solar activity cycles, Astron. Lett. 33, 771-779, 2007.

Tripathy, S.C., Hill, F., Jain, K., Leibacher, J.W. Solar oscillation frequency changes on time scales of nine days, Sol. Phys. 243, 105-120, 2007. 
Ulrich, R.K. Identification of very large scale velocity structures on the solar surface using Mt Wilson synoptic observations, in: Korzennik S.G., Wilson A. (Eds.) Structure and Dynamics of the Interior of the Sun and Sun-Like Stars, SP-418, ESA, Noordwijk, pp. 851-855, 1998.

Ulrich, R.K., Bertello, L. Solar rotation measurements at Mount Wilson over the period 1990-1995, Astrophys. J. 465, L65-L68, 1996.

Wöhl, H., Schmidt, W. Solar equatorial plasma rotation: a comparison of different spectroscopic measurements, Astrono. Astrophys. 357, 763-766, 2000 . 

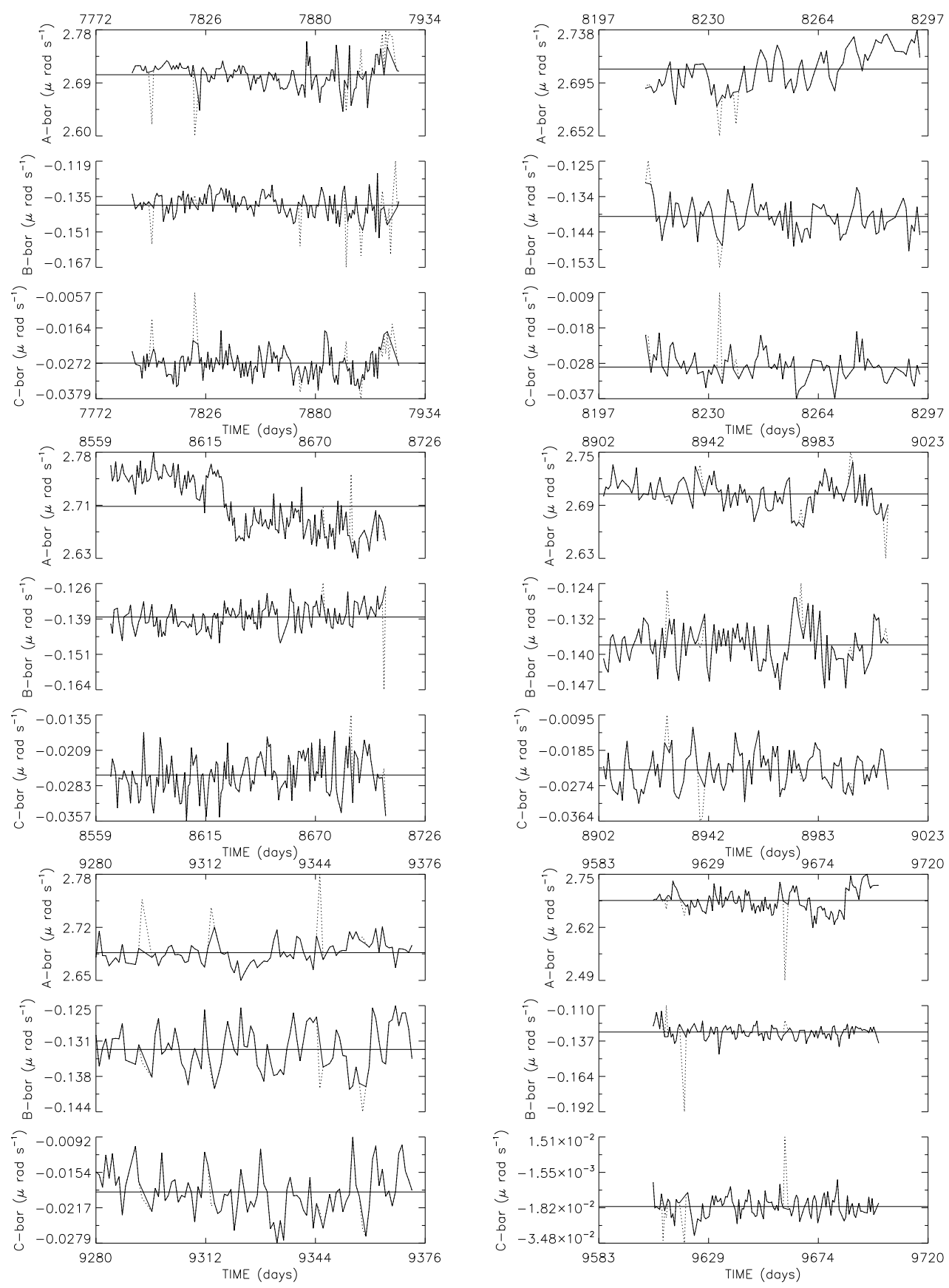

Figure 1: Variations in the $\bar{A}, \bar{B}$ and $\bar{C}$ (cf. Equation (3)) determined from Mt. Wilson velocity data in the time (rotation number) intervals $7772-7934$ (top panel-left), $8197-$ 8297 (top panel-right), 8559-8726 (middle panel-left), 8902-9023 (middle panel-right), 9280-9376 (bottom panel-left), and 9583-9720 (bottom panel-right). The dotted- and solid-curves represent the variations determined from the uncorrected and the corrected data, respectively. The horizontal lines represent the mean values of the corrected data. 


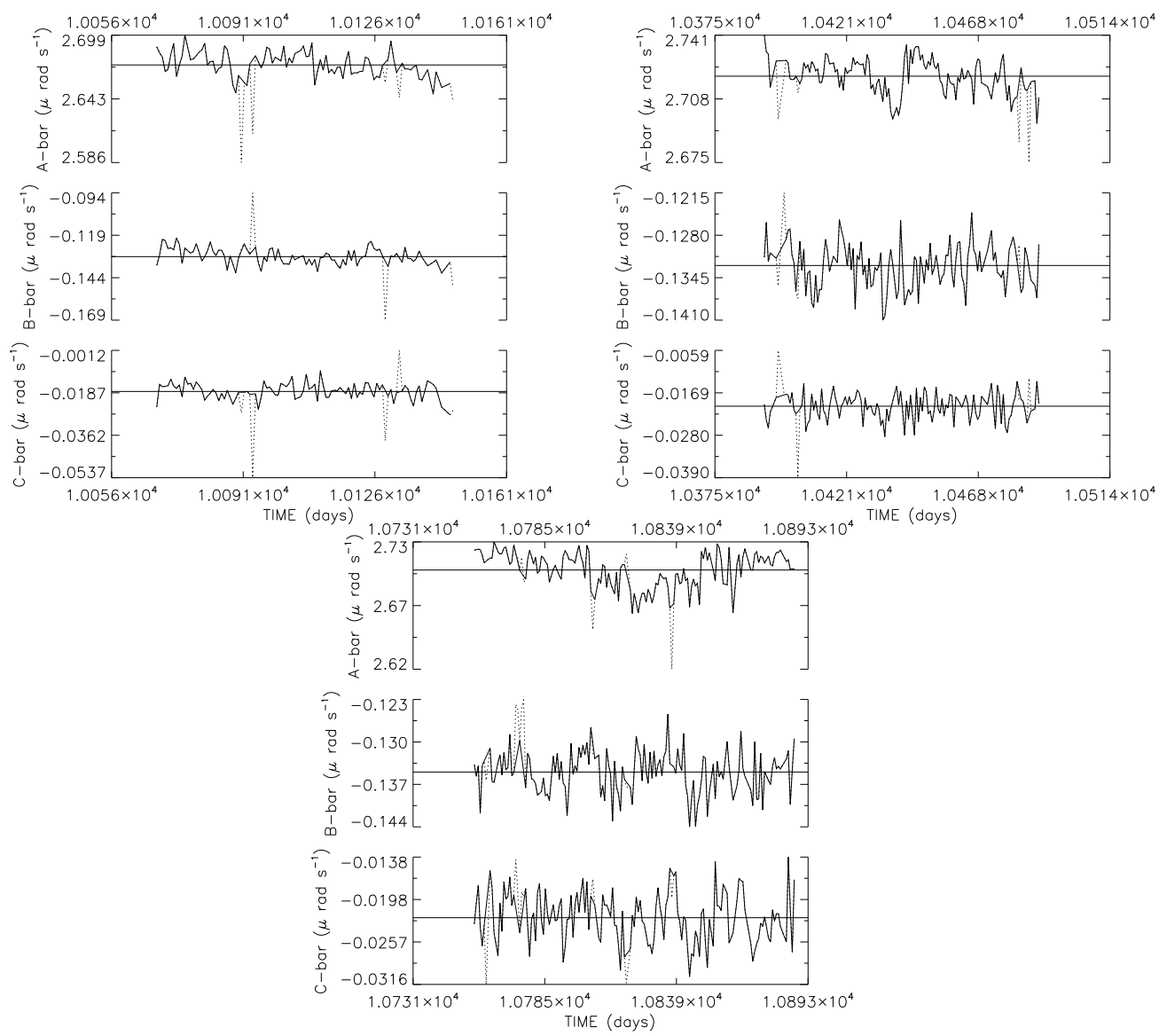

Figure 2: The same as Figure 1, but for the intervals 10056 - 10161 (top panel-left), 1037510514 (top-panel-right), and 10731-10893 (bottom panel). 

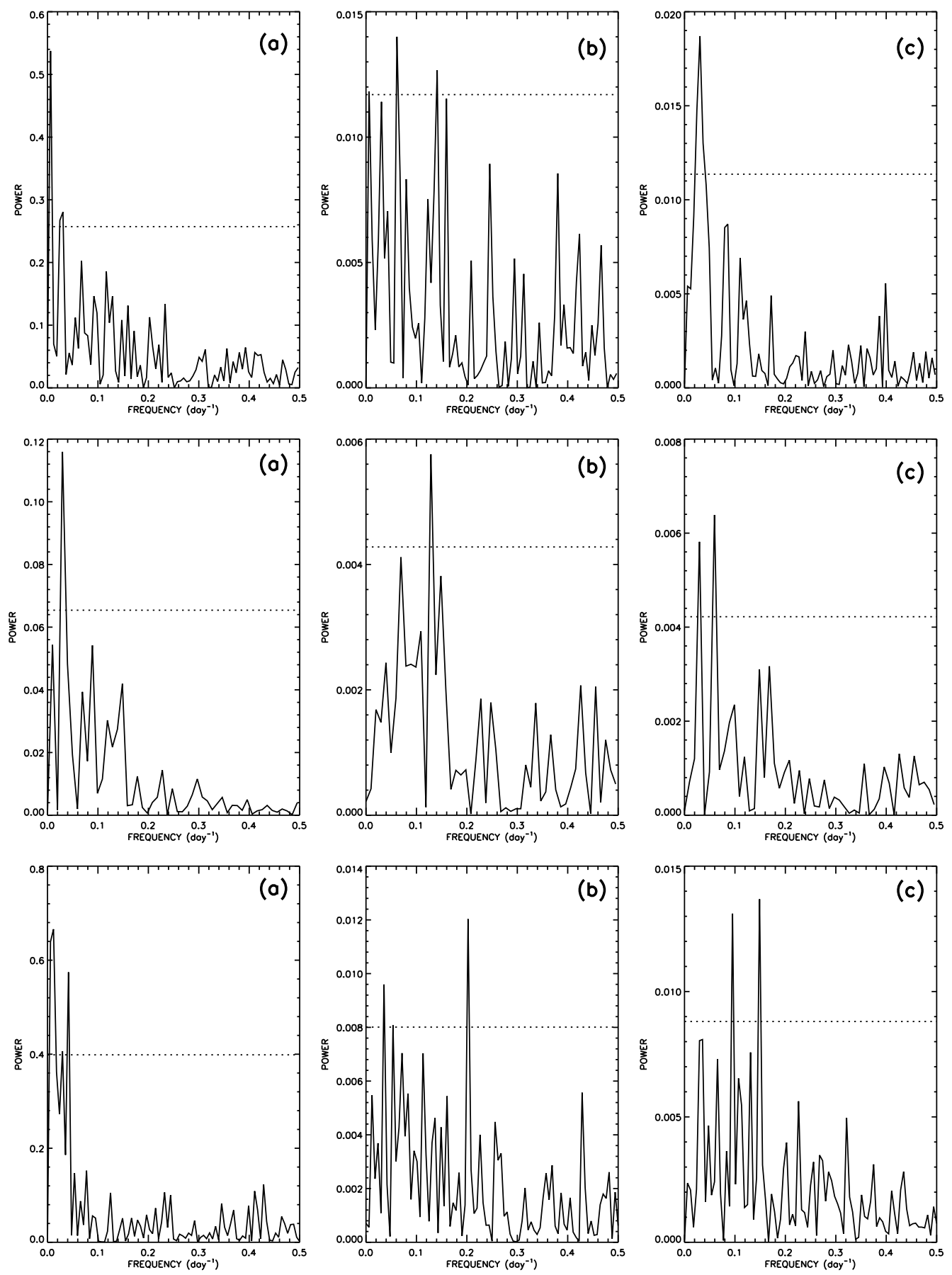

Figure 3: FFT power spectra of (a) $\bar{A}$, (b) $\bar{B}$ and (c) $\bar{C}$ determined from the Mt. Wilson velocity data in the rotation number intervals 7772-7934 (top panel), 8197-8297 (middle panel), and 8559-8726 (bottom panel). The dotted horizontal line represents $99 \%$ confidence level. 

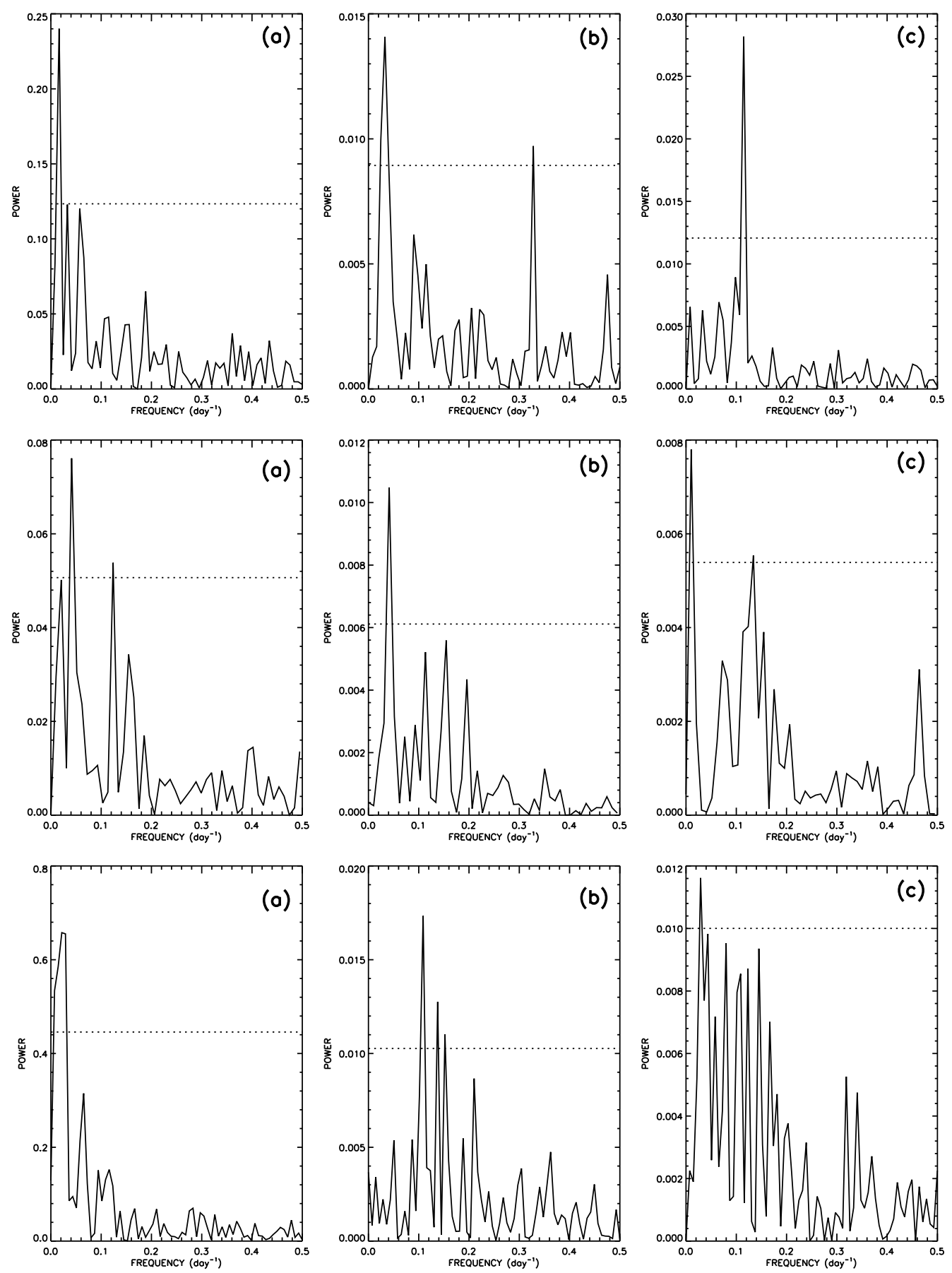

Figure 4: The same as Figure 3, but for the intervals 8902-9023 (top panel), 9280-9376 (middle panel), and 9583-9720 (bottom panel). 

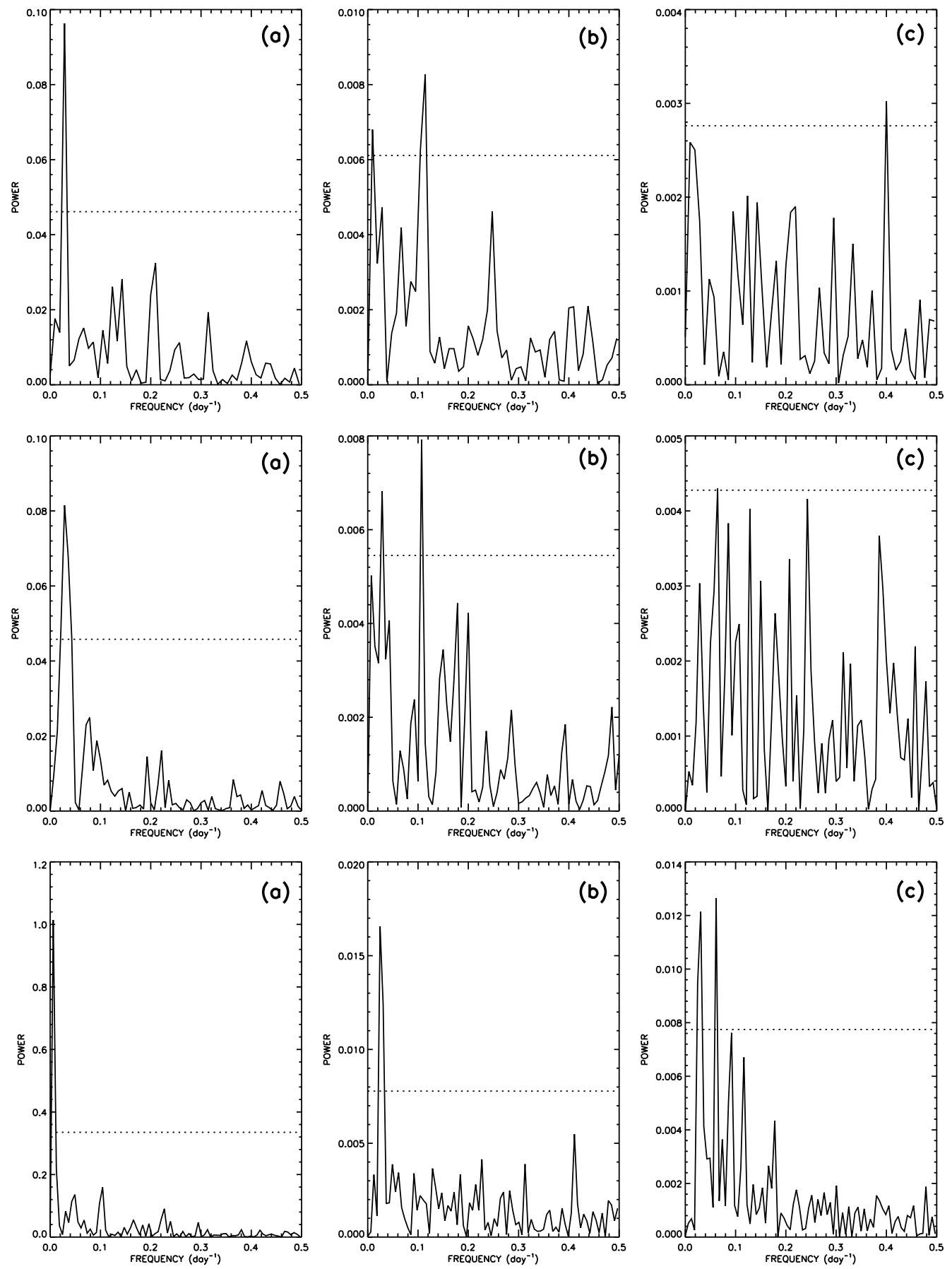

Figure 5: The same as Figure 3, but for the intervals 10056-10161 (top panel), 1037510514 (middle panel), and 10731-10893 (bottom panel). 
Table 1: Periodicities in the variations of $\bar{A}, \bar{B}$, and $\bar{C}$ determined from the daily Mt. Wilson velocity data in different time intervals mentioned in the first column (the corresponding year and number of data points are mentioned within round and square brackets, respectively). The levels of significance (in units of $\sigma$ ) are also given.

\begin{tabular}{|c|c|c|c|c|c|c|}
\hline $\begin{array}{l}\text { Time } \\
\text { Interval } \\
\end{array}$ & $\begin{array}{c}\bar{A} \\
\text { Period (in days) } \\
\end{array}$ & $\sigma$ & $\begin{array}{c}\bar{B} \\
\text { Period (in days) } \\
\end{array}$ & $\sigma$ & $\begin{array}{c}\bar{C} \\
\text { Period (in days) } \\
\end{array}$ & $\sigma$ \\
\hline $\begin{array}{l}7772-7934 \\
(1986),[163]\end{array}$ & $\begin{array}{l}\sim 163 \\
33-41\end{array}$ & $\begin{array}{c}6.2 \\
2.7-2.9\end{array}$ & $\begin{aligned} \sim & \sim 163 \\
\sim & 33 \pm 8 \\
& \sim 16 \\
& 6-7\end{aligned}$ & $\begin{array}{c}3.7 \\
2.4 \\
3.3 \\
2.5-2.9\end{array}$ & $\begin{array}{l}33-41 \\
23-27\end{array}$ & $\begin{array}{l}3.5-4.7 \\
2.4-3.0\end{array}$ \\
\hline $\begin{array}{l}8197-8297 \\
(1987),[101]\end{array}$ & $\begin{array}{l}\sim 101 \\
\sim 34 \\
\sim 11\end{array}$ & $\begin{array}{l}2.0 \\
2.0 \\
2.0\end{array}$ & $\begin{array}{l}\sim 14 \\
6-7\end{array}$ & $\begin{array}{c}2.4 \\
2.2-3.84\end{array}$ & $\begin{array}{r}\sim 34 \\
\sim 17\end{array}$ & $\begin{array}{l}3.8 \\
4.3\end{array}$ \\
\hline $\begin{array}{l}8559-8726 \\
(1988),[168]\end{array}$ & $\begin{array}{l}56-84 \\
\sim 34 \\
\sim 24\end{array}$ & $\begin{array}{l}2.3-4.6 \\
\quad 2.6 \\
3.9\end{array}$ & $\begin{array}{l}\sim 28 \\
\sim 19 \\
\sim 9 \\
\sim 5\end{array}$ & $\begin{array}{l}3.3 \\
2.7 \\
2.1 \\
4.3\end{array}$ & $\begin{array}{c}28-34 \\
\sim 10 \\
6-8\end{array}$ & $\begin{array}{c}2.9 \\
4.3 \\
2.0-4.5\end{array}$ \\
\hline $\begin{array}{l}8902-9023 \\
(1989),[122]\end{array}$ & $\begin{array}{l}\sim 61 \\
\sim 30 \\
\sim 17\end{array}$ & $\begin{array}{l}5.6 \\
2.6 \\
2.5\end{array}$ & $\begin{array}{l}\sim 30 \\
\sim 24 \\
\sim 3\end{array}$ & $\begin{array}{l}4.5 \\
2.4 \\
2.9\end{array}$ & $\sim 9$ & 6.7 \\
\hline $\begin{array}{l}9280-9376 \\
(1990),[97]\end{array}$ & $\begin{array}{l}\sim 48 \\
\sim 24 \\
\sim 8\end{array}$ & $\begin{array}{l}2.5 \\
4.3 \\
2.8\end{array}$ & $\begin{array}{l}\sim 24 \\
\sim 9 \\
\sim 6\end{array}$ & $\begin{array}{l}4.9 \\
2.1 \\
2.3\end{array}$ & $\begin{array}{l}\sim 97 \\
\sim 7\end{array}$ & $\begin{array}{l}4.1 \\
2.7\end{array}$ \\
\hline $\begin{array}{l}9583-9720 \\
(1991),[138]\end{array}$ & $34-69$ & $3.5-4.0$ & $\begin{array}{l}\sim 9 \\
6-7 \\
\sim 4\end{array}$ & $\begin{array}{c}4.9 \\
2.8-3.3 \\
2.0\end{array}$ & $\begin{array}{l}\sim 34 \\
\sim 12 \\
\quad 8-9 \\
\sim 6\end{array}$ & $\begin{array}{l}3.1 \\
2.4 \\
2.0 \\
2.3\end{array}$ \\
\hline $\begin{array}{l}10056-10161 \\
(1992),[106]\end{array}$ & $\sim 35$ & 5.0 & $\begin{array}{l}\sim 35 \\
8-9\end{array}$ & $\begin{array}{c}3.4 \\
2.7-3.8\end{array}$ & $\begin{array}{l}52-105 \\
\sim 2.5\end{array}$ & $\begin{array}{l}2.2-2.3 \\
2.9\end{array}$ \\
\hline $\begin{array}{l}10375-10514 \\
(1993),[140]\end{array}$ & $\sim 35$ & 5.0 & $\begin{array}{l}\sim 140 \\
\sim 35 \\
\sim 9\end{array}$ & $\begin{array}{l}2.3 \\
3.4 \\
4.1\end{array}$ & $\begin{aligned} & \sim 15 \\
& \sim 12 \\
& \sim 8 \\
& \sim 4 \\
& \sim 2.5\end{aligned}$ & $\begin{array}{l}2.6 \\
2.2 \\
2.4 \\
2.5 \\
2.0\end{array}$ \\
\hline $\begin{array}{l}10731-10893 \\
(1994),[163]\end{array}$ & $\sim 163$ & 8.4 & $\sim 32-41$ & $4.5-6.3$ & $\begin{array}{c}\sim 32-41 \\
\sim 16 \\
\sim 11 \\
\quad \sim 9\end{array}$ & $\begin{array}{l}3.3-4.4 \\
4.6 \\
2.5 \\
2.1\end{array}$ \\
\hline
\end{tabular}

